\title{
Carrier Based Single-State PWM Technique for Minimizing Vector Errors in Multilevel Inverters
}

\author{
Nguyen Van Nho*, Quach Thanh Hai*, and Hong-Hee Lee ${ }^{\dagger}$ \\ * Dept. of Electrical and Electronics Eng., HCM University of Technology, Ho Chi Minh, Vietnam \\ ${ }^{\dagger}$ School of Electrical Eng., University of Ulsan, Ulsan, Korea
}

\begin{abstract}
In this paper, a novel analysis of a carrier based PWM method for multilevel inverters is presented. The space vector PWM and carrier based PWM correlations in multilevel inverters are investigated in a nominal two-level switching diagram. The obtained results can be applied to design various carrier PWM techniques. In this paper, a carrier based single-state PWM technique, which reduces the switching number and optimizes the active voltage errors, is presented. This PWM technique can be advantageous if there are a large number of levels. The proposed method is mathematically formulated and demonstrated by simulations and experimental results.
\end{abstract}

Key Words: Minimized voltage error, Multilevel inverter, PWM technique, Single-switching state

\section{INTRODUCTION}

Nowadays, multilevel inverters have become more attractive to researchers and industrial companies. There are two common types, which include NPC and cascaded as shown in Fig.1 and Fig.2 [1]-[5]. Recently, in an effort to reduce hardware construction cost, researchers have attempted to develop prospective hybrid topologies. There are basically three PWM schemes for their control. These include carrier based PWM, space vector PWM and selective harmonics elimination PWM methods [6]-[12]. In comparison to the space vector PWM methods, the carrier based PWM methods can be advantageously utilized in: 1) controlling common mode voltage, 2) controlling complicated inverter topologies such as 4-leg and 5-leg multilevel inverters, and 3) compensating unbalanced dc sources [13], [14]. The space vector modulation method presents a good tool for describing a balanced three phase system. However, its algorithm for implementation is rather difficult, particularly if common mode control is applied to improve PWM performance.

A comprehensive correlation between carrier based PWM and SVPWM has been derived in a recent work. This helps to realize a space vector method with carrier modulation [15]. As a result, a carrier PWM method is more advantageous because its algorithm can be applied to any multilevel inverter and a common mode control is available to improve the modulation performance.

The selected harmonic elimination PWM method (SHE)

Manuscript received Aug. 3, 2009; revised Apr. 24, 2010

† Corresponding Author: hhlee@mail.ulsan.ac.kr

Tel: +82-52-259-2187, Fax: +82-52-259-1686, Univ. of Ulsan

* Dept. of Electrical and Electronics Eng., HCM University of Technology, Vietnam

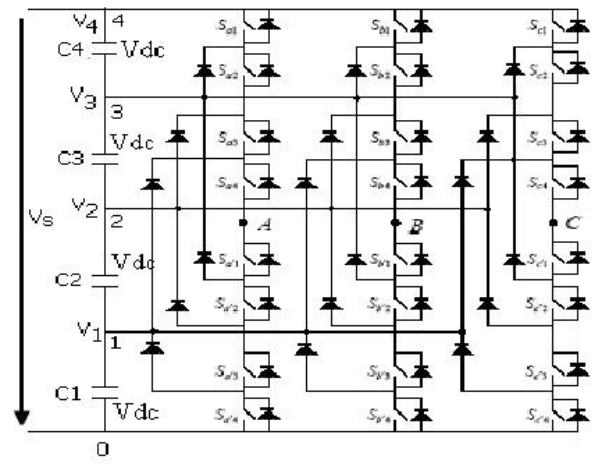

Fig. 1. The 3 phase 5-level NPC inverter.

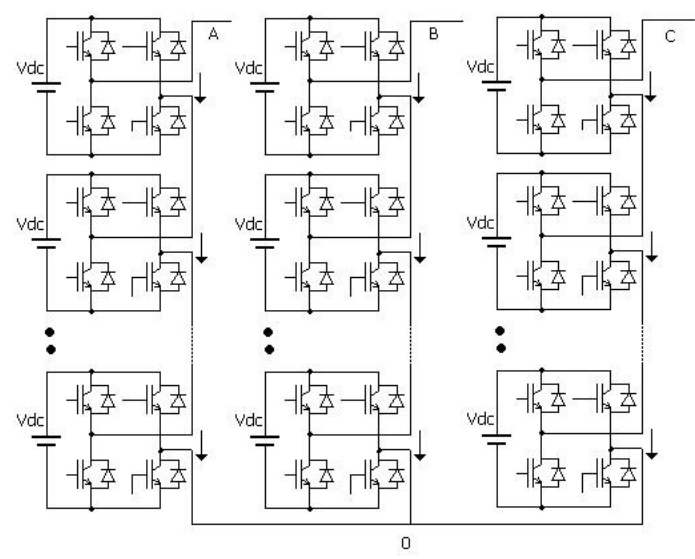

Fig. 2. The 3-phase cascaded multilevel inverter.

is a typical PWM method for reducing switching losses and harmonics distortion. In most cases, only the solutions from 
the offline calculation are valid, particularly for each number of levels. If either the number of levels or the number of the eliminated harmonics is high, finding a solution may be impossible. There are other disadvantages such as the discontinuity of solutions for some output voltage ranges and the limitation on maximum output voltage. To have a similar characteristic, the step pulse modulation approximates the reference fundamental voltage and reduces most of the harmonic content [16]. However, there is still a complex calculation of the commutation angles and a limitation on the maximum output voltage.

A single-state PWM method presents a PWM technique, which produces only one voltage state at the output for a sampling period. In practice, direct torque control and hysteresis current loop control for AC motor drive systems are typical and well-known applications of the single state PWM technique.

As the first approach of a single-state PWM method with the smallest voltage error, a space vector modulation was introduced in recent work [18]. It requires a look-up table, whose data needs to be calculated for each inverter circuit. Another drawback is the limitation on the output voltage. Minimum common mode was set for the implementation but without any discussion on the influence of common mode changing.

In this paper, a carrier based approach to single-state PWM modulation for minimizing voltage errors will be proposed. The deduction is from a NPC inverter analysis, but its proper modification can also be applied to cascade topologies. The carrier based PWM scheme of a multilevel inverter can be centered in a nominal two-level switching state diagram. This makes the PWM study more advantageous and comfortable. The previously described problems can be avoided in the proposed carrier modulation, which is easily realized for any number of levels. As will be shown, the operating range can be extended up to the maximum voltage of the six step mode.

In addition to the fundamental voltage, the common mode voltage control presents a degree of freedom, which can be utilized for setting an optimized modulation, with respect to the THD factor and the switching loss balancing. Besides that, it can be favorably considered for some applications in a four wire unbalanced system. However, that will require further investigation.

\section{TERMINOLOGY AND NOMINAL SWITCHING DIAGRAM FOR MULTILEVEL INVERTERS FOR BALANCED DC VOLTAGE SOURCES}

Assumption: each dc voltage cell is constant and equal to the unit $\left(V_{d c}=1\right)$. Define the reference leg voltages between the outputs $(A, B, C)$ and the dc-neutral point "0" (Fig.1), consisting of the active voltages $v_{x 12}, x=a, b, c$ and the reference common mode $v_{0 \text { ref }}[15]$ as:

$$
v_{x r e f}=v_{x 12}+v_{0 r e f} \text {. }
$$

Or in the vector form as:

$$
\vec{v}_{r e f}=\vec{v}_{12}+v_{0 r e f} \vec{I}
$$

where $\vec{v}_{r e f}=\left[v_{\text {aref }}, v_{\text {bref }}, v_{\text {cref }}\right]^{T}, \vec{I}=[1,1,1]^{T}$ is a unit vector, and:

$$
\vec{v}_{12}=\left[v_{a 12}, v_{b 12}, v_{c 12}\right]^{T} .
$$

The active voltages or the fundamental voltages can be described as follows:

$$
\begin{aligned}
& v_{a 12}=v_{\text {ref }} \cos \theta \\
& v_{b 12}=v_{\text {ref }} \cos (\theta-2 \pi / 3) ; \\
& v_{c 12}=v_{\text {ref }} \cos (\theta-4 \pi / 3)
\end{aligned}
$$

where $V_{\text {ref }}$ and $\theta$ are the amplitude and the phase argument of the reference voltage vector.

Define Max and Min as the maximum and minimum values from the three phase voltages as:

$$
\begin{aligned}
& \operatorname{Max}=\operatorname{Max}\left(v_{a 12}, v_{b 12}, v_{c 12}\right) \\
& \operatorname{Min}=\operatorname{Min}\left(v_{a 12}, v_{b 12}, v_{c 12}\right)
\end{aligned}
$$

The reference common mode voltage can be proposed in the range of $v_{0 M a x}$ and $v_{0 M i n}$ as:

$$
\begin{aligned}
& v_{0 M a x}=(n-1)-\operatorname{Max} \\
& v_{0 \text { Min }}=- \text { Min }
\end{aligned}
$$

The active Low $L_{(x)}$ and High $H_{(x)}$ levels correspond to the two dc voltages nearest to the reference leg voltage $v_{x r e f}$ as shown in Fig.3(a). This can be described as:

$$
\begin{aligned}
& L(x)=\left\{\begin{array}{ccc}
n_{(x)} & \text { if } & 0 \leq v_{\text {ref }}<(n-1) \\
n_{(x)}-1 & \text { if } & v_{\text {ref }}=(n-1)
\end{array}\right. \\
& H_{(x)}=L_{(x)}+1 \\
& \text { where } n_{(x)}=\operatorname{Int}\left(v_{\text {xref }}\right) ; x=a, b, c .
\end{aligned}
$$

The components of the vector $\vec{L}=\left[L_{a}, L_{b}, L_{c}\right]^{T}$ present three lower levels of the leg voltages in the considered switching state sequence.

Nominal switching time diagram: Investigation of the commutation process in a triangle period can be followed in Fig.3(b). The commutation instants are determined by comparing the active carrier waves with the modulating signals $\xi_{x}, x=a, b, c$, defined as:

$$
\xi_{x}=v_{x r e f}-L(x) ; 0 \leq \xi_{x} \leq 1 ;
$$

$$
\begin{aligned}
& \text { or } \\
& \qquad \vec{\xi}=\vec{v}_{r e f}-\vec{L} \\
& \text { where } \vec{L}=\left[L_{a}, L_{b}, L_{c}\right]^{T} \text {. } \\
& \text { Nominal switching state sequence: As seen in Fig.3(b) the } \\
& \text { switching time diagram for a multilevel inverter is similar to } \\
& \text { that for a two-level inverter. Define the nominal switching } \\
& \text { states as: }
\end{aligned}
$$




$$
\begin{aligned}
& \vec{s}_{1}=[0,0,0]^{T} \\
& \vec{s}_{2}=\left[S_{2 a}, S_{2 b}, S_{2 c}\right]^{T} \\
& \vec{s}_{3}=\left[S_{3 a}, S_{3 b}, S_{3 c}\right]^{T} \\
& \vec{s}_{4}=[1,1,1]^{T} .
\end{aligned}
$$

The first and the last states $\vec{s}_{1} ; \vec{s}_{4}$ remind the two zero redundant states in a two-level inverter. The two remaining states $\vec{s}_{2} ; \vec{s}_{3}$ can be determined from the relative positions of the signals $\xi_{x}$ and the two nearest corresponding dc levels.

Define the maximum, medium and minimum values of the signals $\xi_{a}, \xi_{b}, \xi_{c}$ as:

$$
\begin{aligned}
& \xi_{\text {Max }}=\operatorname{Max}\left(\xi_{a}, \xi_{b}, \xi_{c}\right) \\
& \xi_{\text {Mid }}=\operatorname{Mid}\left(\xi_{a}, \xi_{b}, \xi_{c}\right) \\
& \xi_{\text {Min }}=\operatorname{Min}\left(\xi_{a}, \xi_{b}, \xi_{c}\right) .
\end{aligned}
$$

The components of the vectors $\vec{s}_{2} ; \vec{s}_{3}$ can be derived as follows:

$$
\begin{aligned}
& s_{2 x}=\left\{\begin{array}{ll}
1 & \text { if } \\
0 & \text { else }
\end{array} \quad \xi_{x} \geq \xi_{\text {Max }}\right. \\
& s_{3 x}=\left\{\begin{array}{ll}
1 & \text { if } \\
0 & \text { else }
\end{array} \quad \xi_{x} \geq \xi_{\text {Mid }}\right.
\end{aligned}
$$

The switching state sequences in a multi-carrier multilevel inverter $\vec{S}_{1}, \vec{S}_{2}, \vec{S}_{3}, \vec{S}_{4}$ can be easily deduced by:

$$
\vec{S}_{j}=\vec{L}+\vec{s}_{j}
$$

Conventional space vector modulation is based on the implementing of the three nearest vectors for the corresponding switching time duties as $\left(K_{14}, K_{2}, K_{3}\right)$, respectively and is described as:

$$
\vec{v}_{r e f}=K_{1} \vec{S}_{1}+K_{2} \vec{S}_{2}+K_{3} \vec{S}_{3}+K_{4} \vec{S}_{4}
$$

For example, the vector $\vec{S}_{1}=[2,1,0]^{T}$ corresponds to the three phase leg voltages as $2 V_{d c}, V_{d c}$ and 0 . It can be deduced from Fig. 3 by:

$$
\begin{aligned}
& K_{1}=1-\xi_{\text {Max }} ; K_{2}=\xi_{M a x}-\xi_{M i d} \\
& K_{3}=\xi_{\text {Mid }}-\xi_{\text {Min }} ; K_{4}=\xi_{\text {Min }} \\
& K_{14}=1-\xi_{\text {Max }}+\xi_{\text {Min }} \\
& K_{1}+K_{2}+K_{3}+K_{4}=1 .
\end{aligned}
$$

\section{PROPOSED SINGLE STATE PWM METHOD}

The principle behind the single-state PWM method: The proposed modulation can be described as a further step of the conventional SVPWM defined by (13). As shown in Fig.5, the PWM generator will set only one switching state for an entire sampling period:

$$
\vec{v}_{\text {ref }}^{\prime}=\vec{S}_{j} ; j \in 1,2,3,4
$$

(a)

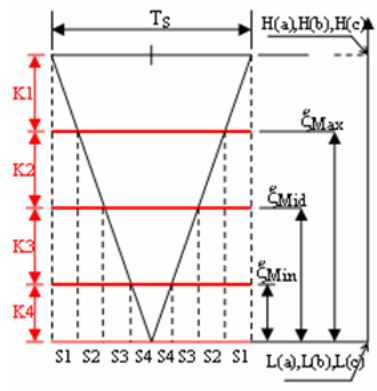

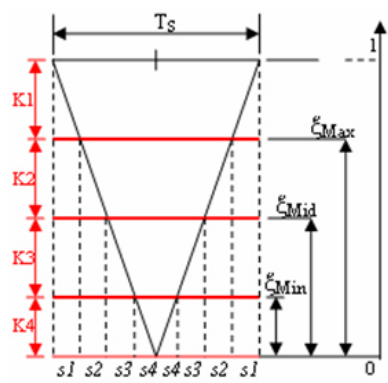

(b)
Fig. 3. (a) Switching time diagram deduced in (b) new defined coordinates and (b) nominal switching time diagram.

The selected vector can be any of the 4 relevant switching states $\vec{S}_{1}, \vec{S}_{2}, \vec{S}_{3}$ and $\vec{S}_{4}$. The remaining states from the switching sequence are rejected.

The proposed modulation for minimized voltage error is based on the implementation of the nearest vector. The condition for selecting the switching state is shown in Fig.4(a) and (b) and can be described as:

$$
\vec{v}_{r e f}^{\prime}=\vec{S}_{j} \text { for }|\vec{e}|=\left|\vec{v}_{r e f}-\vec{S}_{j}\right| \rightarrow \text { Min }
$$

For example, the switching state $\vec{S}_{3}$ is implemented because the vector $\vec{V}_{3}$ is closest to the reference.

Determination of the nearest vector: two possible redundant states generate the same voltage vector, and they can be presented by one pivot vector, whose resulting switching time duty is equal to the sum of their switching time duties.

Each pivot vector $\vec{V}_{j}$ characterizes a certain contribution to the reference with its time duty $\vec{K}_{j}$. The influence is stronger if it is closer to the reference vector. It can be easily deduced that from the space vector modulation, the vector nearest to the reference vector will have the highest value of switching time duty. As a result, for implementing the proposed PWM method, it is necessary to set the reference vector of the maximum time duty, derived in the conventional PWM method, i.e.:

$$
K_{j}=K_{M a x}=\operatorname{Max}\left(K_{14}, K_{2}, K_{3}\right) .
$$

For any reference vector, the influence of the individual pivot vectors on the function of $K_{\text {Max }}$ defines three symmetrical sub-areas $A_{j}, \mathrm{j}=1,2,3$ in the triangle, as shown in Fig.4(a). For example, if the reference vector $\vec{V}_{\text {ref }}$ is located in the subarea A3, where the condition $K_{3}=K_{\text {Max }}$ is valid, the active error will be minimized if the output voltage is produced by the vector $\vec{v}_{\text {ref }}^{\prime}=\vec{S}_{3}$ (Fig.4(b)). Particularly, if it happens that $K_{\text {Max }}=K_{14}$ while both of the vectors $\vec{S}_{1}$ and $\vec{S}_{4}$ have the same error $\left|\vec{e}_{12}\right|$.

The criterion for a minimized offset error $e_{0}$ can be taken into account for setting the reference $\vec{V}_{r e f}^{\prime}$. For example, the vector $\vec{S}_{1}$ is selected if:

$$
\left|\operatorname{Offset}\left(\vec{S}_{1}-\vec{V}_{\text {ref }}\right)\right|<\left|\operatorname{Offset}\left(\vec{S}_{4}-\vec{v}_{r e f}\right)\right|
$$

or, if after derivation, the condition is: 


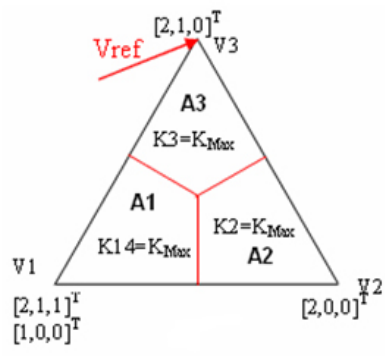

(a)

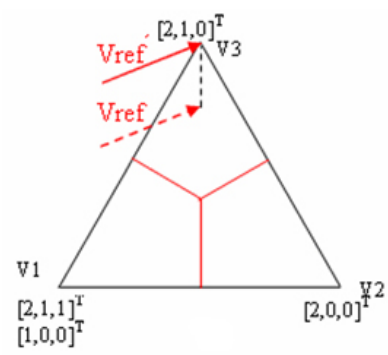

(b)
Fig. 4. The single-state PWM method with a minimized voltage error (a) the function $K_{\max }$ in a triangle area, and (b) explanation of the principle.

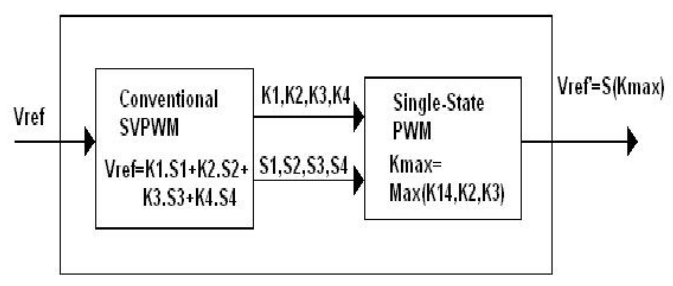

Fig. 5. The principle of the single-state PWM generator.

$$
K_{2}+2 K_{3}+3 K_{4}<1.5
$$

The algorithm for the minimum voltage error based singlestate PWM can be deduced in relation to the switching time duties as described in Table 1. If the reference vector appears at the center of the triangle, i.e. $K_{14}=K_{2}=K_{3}=1 / 3$, the active error can achieve a maximum value of:

$$
e_{12 \operatorname{Max}}=2 /(3 \sqrt{3})
$$

TABLE I

ALGORITHM OF THE SINGLE-STATE PWM METHOD

\begin{tabular}{|c|c|}
\hline $\begin{array}{c}\text { Conditions in the } \\
\text { conventional PWMs }\end{array}$ & $\begin{array}{c}\text { Selected vectors and the } \\
\text { error } e_{12}\end{array}$ \\
\hline$K_{14}>K_{2} ; K_{14}>K_{3}$ & $\vec{v}_{r e f}^{\prime}=\vec{S}_{1}$ \\
$K_{2}+2 K_{3}+3 K_{4}<1.5$ & $e_{12}=\frac{2}{3} \sqrt{K_{2}^{2}+K_{3}^{2}+K_{2} K_{3}}$ \\
\hline$K_{2}>K_{3} ; K_{2}>K_{14}$ & $\vec{v}_{r e f}^{\prime}=\vec{S}_{2}$ \\
& $e_{12}=\frac{2}{3} \sqrt{K_{14}^{2}+K_{3}^{2}+K_{14} K_{3}}$ \\
\hline$K_{3}>K_{2} ; K_{3}>K_{14}$ & $\vec{v}_{r e f}^{\prime}=\vec{S}_{3}$ \\
& $e_{12}=\frac{2}{3} \sqrt{K_{14}^{2}+K_{2}^{2}+K_{14} K_{2}}$ \\
\hline$K_{1}>K_{2} ; K_{14}>K_{3}$ & $\vec{v}_{r e f}^{\prime}=\vec{S}_{4}$ \\
$K_{2}+2 K_{3}+3 K_{4}>1.5$ & $e_{12}=\frac{2}{3} \sqrt{K_{2}^{2}+K_{3}^{2}+K_{2} K_{3}}$ \\
\hline
\end{tabular}

\section{Over-modulation in the single-state PWM method}

For the single-state PWM method, the control characteristic is non-linear. The low order harmonics appear for any modulation index. This differs from the conventional PWM methods, and the term of over-modulation in the proposed method losses its original meaning. However, over-modulation can be seen as an approach to extend the reference fundamental voltage

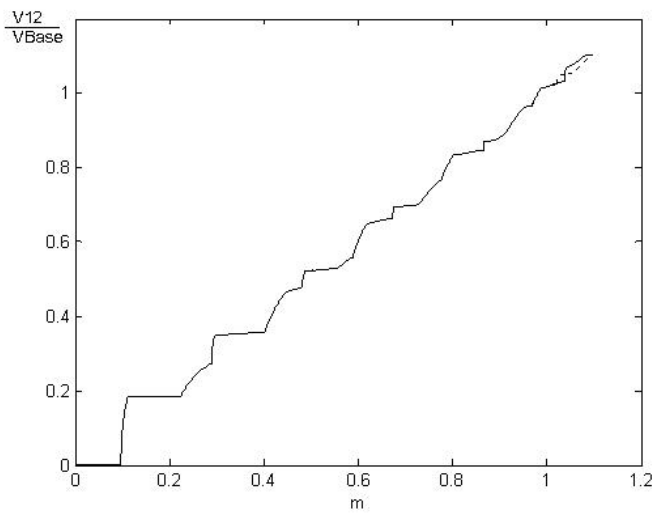

Fig. 6. The control characteristic of 7-level inverter.

$V_{(1) m r e f}$ in the range of $\left(V_{s} / \sqrt{3}, 2 V_{s} / \pi\right)$, where $\mathrm{Vs}$ is the total voltage on the dc side. The active voltages in single-state over-modulation can be deduced from the control principle between the two-limit trajectories [15], for which the signals $v_{x 12, m}$ related to the modulating index of $\mathrm{m}\left(m_{A} \leq m \leq m_{B}\right)$ can be deduced from the active signals of the corresponding limit modulation indexes of $m_{A}, m_{B}$ as:

$$
\begin{aligned}
& v_{x 12, m}=(1-\eta) v_{x 12, A}+\eta v_{x 12, B} \\
& \text { where } \eta=\left(m-m_{A}\right) /\left(m_{B}-m_{A}\right) .
\end{aligned}
$$

Compared to the limit deduced in [2], the proposed overmodulation method has a wider range and it can reach a maximum modulation index up to that of the six-step method. A simple two-mode over-modulation can be applied, using the three limit modulation indexes of $1,1.055$ and 1.1.

The control characteristic

A diagram of the nonlinear control characteristic of a sevenlevel inverter is calculated and drawn in Fig.6.

Single-state PWM demonstrates its benefit in reducing the switching losses for a higher number of levels and for higher modulation indexes. The existing error for the non-linearity of the control characteristic in an open-loop control may be reduced with an increase in the number of levels, or it may be compensated for in a close-loop control system.

Optimized switching loss single-state PWM method

The relationship between the number of switchings $(\mathrm{N})$ per period of the output voltage and the THD factor was deduced for 4 PWM cases from Table 1. These are: 1) Conventional PWM with minimum common mode; 2) Conventional PWM with medium common mode; 3) Single-state PWM with minimum common mode and 4) Single-state PWM with medium common mode.

The number of switchings for the single-state PWM method depends on the modulation index, the common mode voltage and the number of levels. To compare the switching loss, the minimum and the medium common mode PWM methods have been investigated. A detailed study for a 7-level inverter is shown in Fig.7. The second method has a lower number of switchings for the narrow modulation index range of $(0.68<\mathrm{m}<0.72)$, while the first one is advantageous for a 


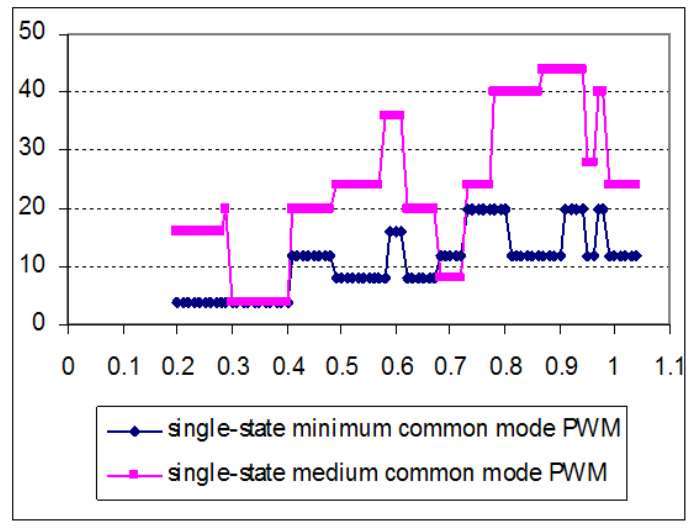

Fig. 7. Single-state PWM method in 7-level inverter - diagrams of number of switching for two different common mode functions.

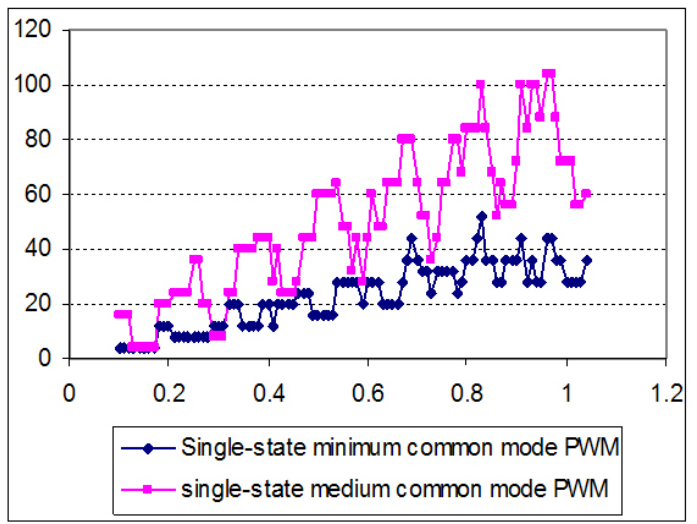

Fig. 8. Single-state PWM methods in a 15-level inverter - diagrams of the number of switching for two different common mode functions.

smaller number of switchings for nearly the entire modulation index range $(\mathrm{m}<0.68)$ and $(0.72<\mathrm{m}<1.1)$. A combination of both methods can set up an optimized PWM method. Furthermore, the minimum common mode method introduces a better distribution of switching loss among the switching devices. It is possible to have a conclusion for a 15-level inverter topology that is similar to the way shown in Fig.8.

\section{Harmonic distortion factor}

Comparing the THD factors of a conventional PWM method and the single-state PWM for an 11-level inverter, the first approach causes distortion given mainly by carrier sideband harmonics, while the second one mostly gives rise to the loworder harmonics. A detailed study of the characteristics of the 4 mentioned methods was realized and shown in Table 1. For an output frequency of $50 \mathrm{~Hz}$, a modulation index of $\mathrm{m}=1$, and a carrier frequency $f_{s w}$ of $1080 \mathrm{~Hz}$, the conventional PWM modulation for the minimum common mode gives 28 switchings. For comparison, the single-state PWM has the same number of switchings, but it gains a reduction in the THD factor $(4.1 \%$ against $5.7 \%)$. In principle, there is no difference in the THD factors from both single-state PWM methods. For the entire modulation index, the THD factor diagrams of the 11-level inverter for the corresponding cases were drawn in Fig.9. The carrier frequencies of the first two methods were selected to be $600 \mathrm{~Hz}$.
TABLE II

COMPARISON OF THD FACTORS AND NUMBER OF SWITCHING FOR DIFFERENT PWM METHODS

\begin{tabular}{|c|c|c|c|c|c|c|c|}
\hline $\mathrm{m}$ & 0.4 & 0.5 & 0.6 & 0.7 & 0.8 & 0.9 & 1 \\
\hline$f_{s w}$ & 300 & 300 & $450(300)$ & 600 & 720 & 600 & $900(1080)$ \\
\hline $\mathrm{N} 1$ & 10 & 14 & 20 & 26 & 28 & 20 & $24(28)$ \\
\hline THD1 & 15.2 & 9.3 & 8.7 & 9.4 & 7.0 & 5.9 & $5.4(5.7)$ \\
\hline $\mathrm{N} 2$ & 10 & 18 & $24(14)$ & 30 & 33 & 30 & $38(45)$ \\
\hline THD2 & 13.3 & 12.0 & $9.2(8.4)$ & 8.5 & 6.7 & 5.9 & $5.6(5.8)$ \\
\hline N3 & 8 & 12 & 20 & 24 & 28 & 20 & 28 \\
\hline THD3 & 10.2 & 7.7 & 6.3 & 5.9 & 5.0 & 4.4 & 4.1 \\
\hline N4 & 20 & 40 & 24 & 60 & 32 & 64 & 52 \\
\hline THD4 & 10.2 & 7.7 & 6.3 & 5.9 & 5.0 & 4.4 & 4.1 \\
\hline
\end{tabular}

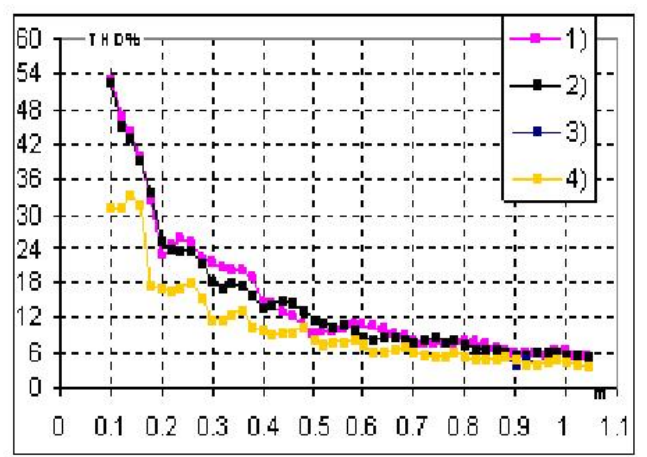

Fig. 9. The 11-level inverter - diagrams of THD factors for conventional PWM methods, $f_{s w}=600 \mathrm{~Hz}$ with 1) minimum common mode; 2) medium common mode. The THD diagrams for single-state PWM methods with 3) minimum common mode and 4) medium common mode.

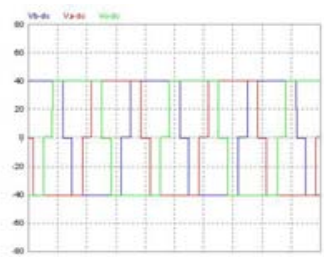

(a)

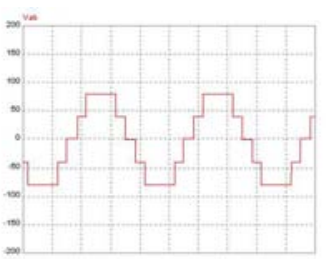

(c)

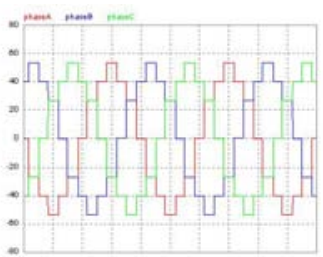

(b)

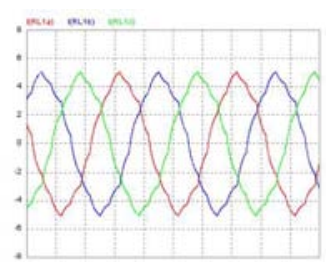

(d)
Fig. 10. Simulation results for $m=0.4$ - diagrams of (a) the inverter voltage, (b) the load voltage, (c) the line-line voltage and (d) the load currents.

The optimized single-state PWM algorithm was advantageously applied to a very high level inverter. In this case, the control linearity can be approximately extended to both the limits of a near zero modulation index and the maximum modulation index of the six-step mode. The THD factor is negligible. In a 31 -level inverter, for $\mathrm{m}=0.2$, the THD is $6.32 \%$ while for $m>0.3$, the THD is less than $4.35 \%$.

\section{SIMULATION AND EXPERIMENTAL RESULTS}

To validate the theoretical analysis, simulations and experiments were implemented for a hybrid and cascaded sevenlevel inverter. Each phase consists of two-H bridge inverters 


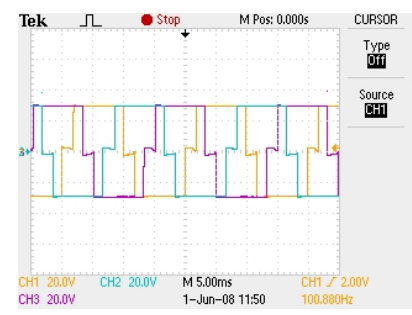

(a)

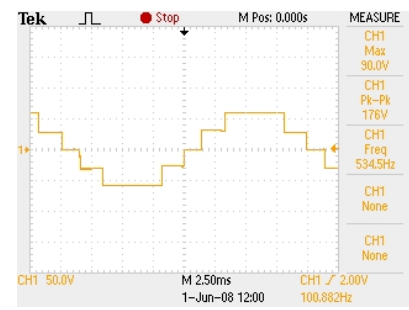

(c)

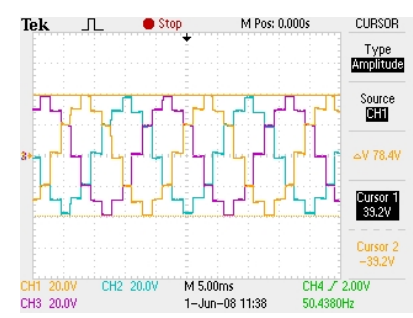

(b)

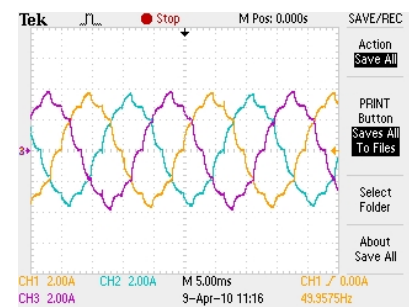

(d)
Fig. 11. The experimental results for $m=0.4$ - diagrams of (a) the inverter voltage, (b) the load voltage, (c) the line-line voltage and (d) the load currents.

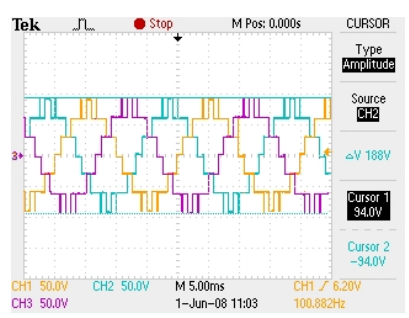

(a)

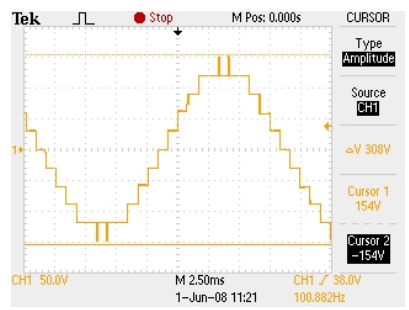

(c)

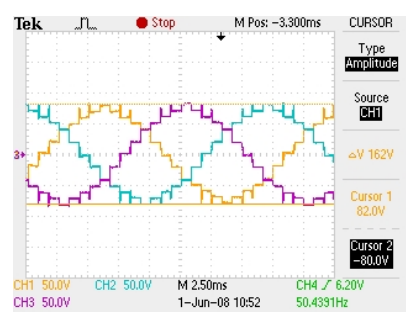

(b)

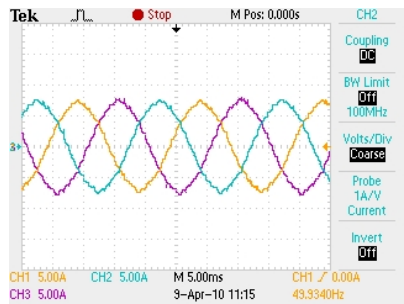

(d)

Fig. 13. The experimental results for $m=0.85$ - diagrams of (a) the inverter voltage, (b) the load voltage, (c) the line-line voltage and (d) the load currents.

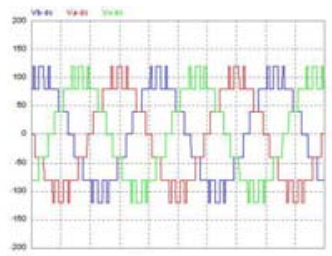

(a)

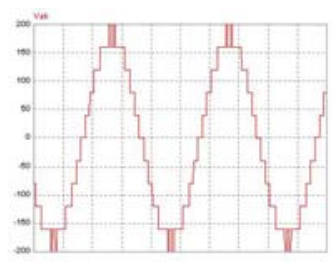

(c)

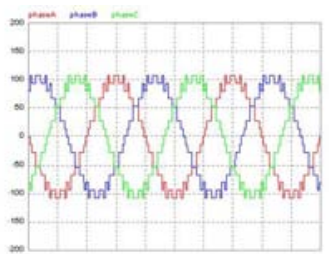

(b)

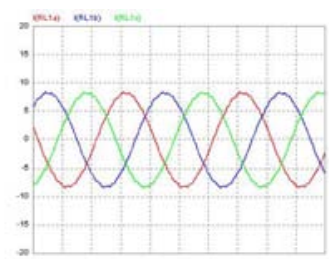

(d)

Fig. 12. Simulation result for $m=0.85$ - diagrams of (a) the inverter voltage, (b) the load voltage, (c) the line-line voltage and (d) the load currents.

in series. The first H-bridge is supplied by a DC source of $40 \mathrm{Vdc}$ and the second is supplied by a source of $80 \mathrm{Vdc}$. A three-phase RL load is set as $R=10 \Omega, L=180 \mathrm{mH}$. The simulations were implemented using PSIM 6.0. The diagrams of the leg voltage, the phase voltage, the line-line voltage and the current were calculated and drawn in Fig.10, 12, 14 and 16. They correspond to several modulation indexes as $0.4,0.85$ and 1 in under-modulation and to 1.04 in overmodulation. The single-state PWM method is advantageously operated in a medium and high modulation index, where the harmonic amplitude distortion is normally small and the control characteristic is nearly linear. The hardware parameters are as follows: an IGBT FG60N100BNTD H20, an IGBT driver with a photo-couple PC123, a dead-time of $3 \mu S$ set by hardware, a measuring oscilloscope TDS2014b, a control kit eZdsp TMS320F2812 and software CCStudio V3.1. The switching frequency was selected as $1050 \mathrm{~Hz}$. The diagrams of the leg voltage, the phase-load voltage, the line-line voltage

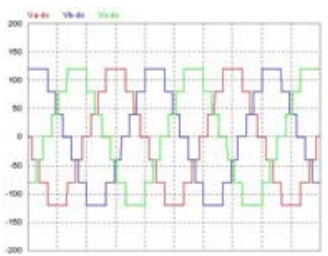

(a)

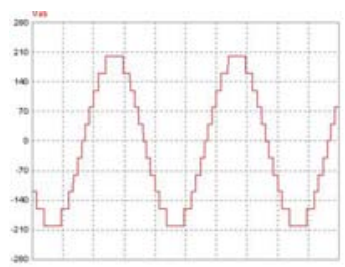

(c)

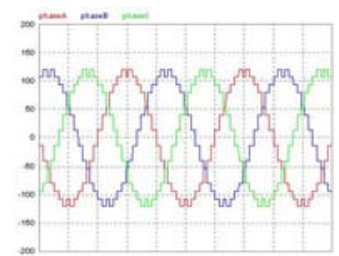

(b)

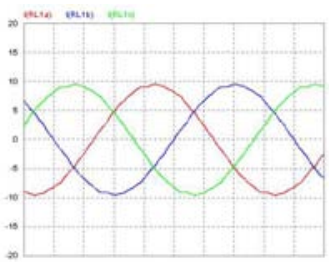

(d)
Fig. 14. The simulation results for $m=1$ - diagrams of (a) the inverter voltage, (b) the load voltage, (c) the line-line voltage and (d) the load currents.

and the phase current for modulation indexes of $0.4,0.85,1$ and 1.04 were measured and shown in Fig.11, 13, 15 and 17. The current quality was evaluated through FFT analysis as shown in Fig.18 and 19.

The obtained experimental results, which are similar to the simulation results, prove the effectiveness of the proposed PWM algorithm. It is clearly seen that the output voltage/current is of a lower quality for a low modulation index. The harmonic distortion can be improved for the higher voltage range. The harmonic distortion of single-state PWM in the over-modulation range is similar to that of the conventional PWM methods. The situation is still good for the lower range (near $\mathrm{m}=1$ ). At around six-step mode, the harmonic content is abruptly increased. 


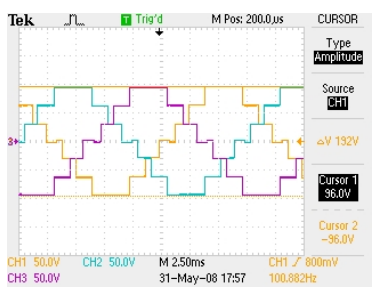

(a)

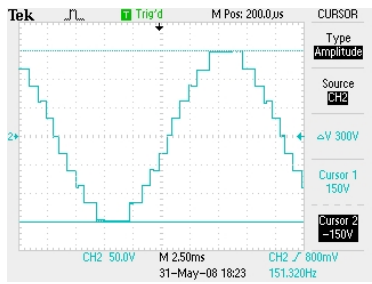

(c)

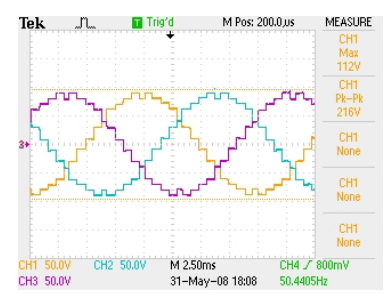

(b)

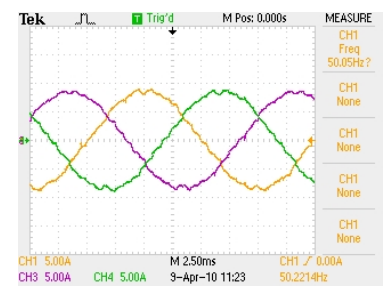

(d)
Fig. 15. Experimental result for $m=1$ - diagrams of (a) the inverter voltage (b) the load voltage, (c) the line-line voltage and (d) the load currents.

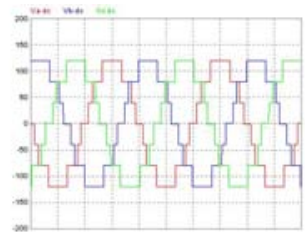

(a)

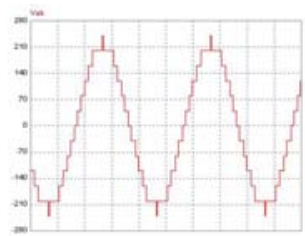

(c)

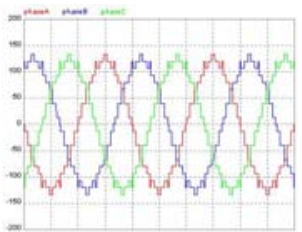

(b)

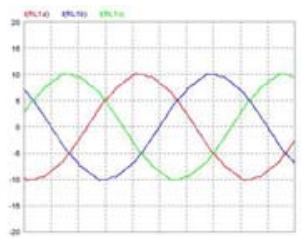

(d)
Fig. 16. The simulation results for $m=1.04$ - diagrams of (a) the inverter voltage, (b) the load voltage, (c) the line-line voltage and (d) the load currents.

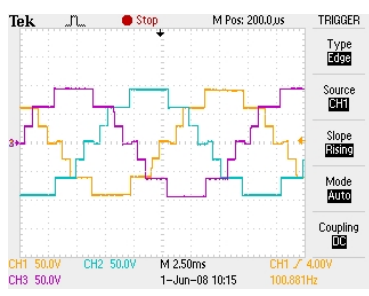

(a)

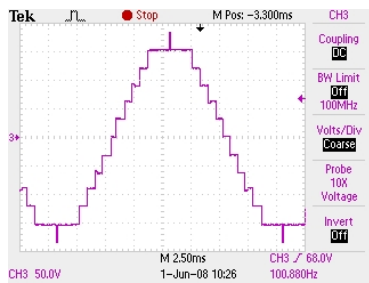

(c)

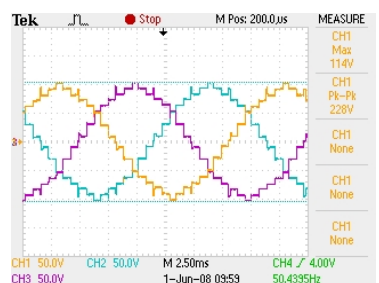

(b)

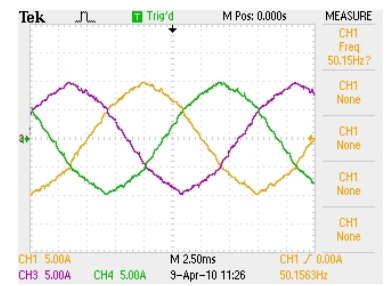

(d)
Fig. 17. The experimental results for $m=1.04$ - diagrams of a) the inverter voltage, b) the load voltage, c) the line-line voltage and d) the load currents.

\section{CONCLUSIONS}

In this paper, a new single-state PWM method with minimum voltage error for multilevel inverters has been proposed.

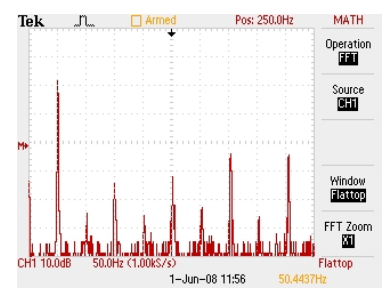

(a)

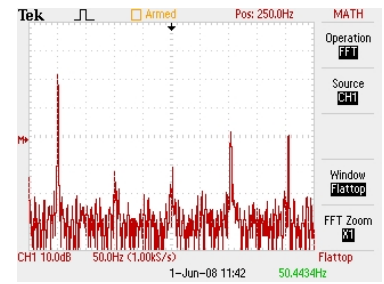

(c)

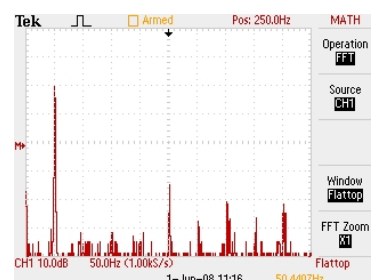

(b)

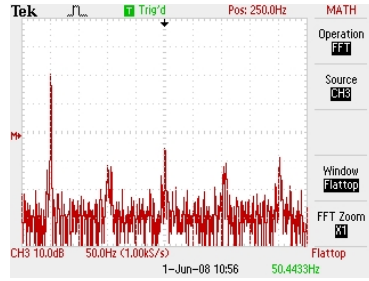

(d)
Fig. 18. Experimental results - diagrams of FFT analysis of the load current and the voltage for $\mathrm{m}=0.4$ (diagrams (a) and (c)) and $\mathrm{m}=0.85$ (diagrams (b) and (d)).

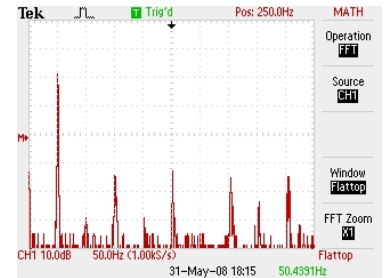

(a)

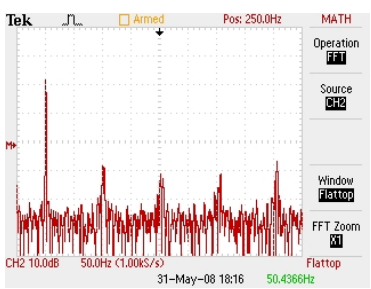

(c)

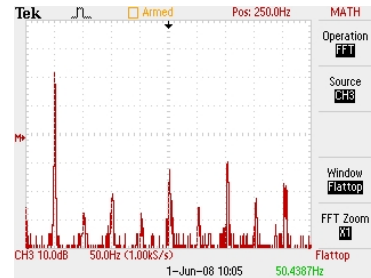

(b)

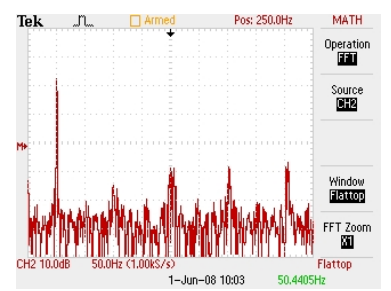

(d)
Fig. 19. Experimental results - diagrams of FFT analysis of the load current and the voltage for $\mathrm{m}=1$ (diagrams (a) and (c)) and $\mathrm{m}=1.04$ (diagrams (b) and (d)).

Using the proper voltage model of an inverter circuit, it can transform the switching state diagram of multi-carrier modulation into that of a conventional two-level inverter. The parameters from the deduced nominal switching diagram can be used to propose a single-state PWM with minimum voltage error. The proposed method has been verified by simulation and experimental results. The benefit of the method can be more effective if a large number of levels is considered. The non-linear control characteristic becomes negligible with an increase in the number of levels. As a result, the carrier based single-state PWM method with the smallest common mode presents a preferable PWM solution for high power and high number-level inverters. For a small number of levels, the drawback of the single state PWM for small voltages can be solved with a combined PWM algorithm. 


\section{REFERENCES}

[1] J.Rodriguez, J. S. Lai and F. Z. Peng, "Multilevel inverters: a survey of topologies, controls and applications," IEEE Trans. Industrial Electronics, Vol. 49, No. 4, pp.724-738, Aug. 2002.

[2] Jose Rodríguez, Luis Morán, Pablo Correa and Cesar Silva, "A vector control technique for medium-voltage multilevel inverters," IEEE Trans. On Industrial Electronics, Vol. 49, No. 4, Aug. 2002.

[3] G. Carrara, S.Gardella,M. Marchesoni,R. Salutari, and G. Sciutto, "A new multilevel PWM method- a theoretical analysis," IEEE Trans. On Power Electronics, Vol.7, No.3, pp.497-505, Jul. 1992.

[4] S. Wei, B.Wu, F. L. and C. Liu, "A general space vector PWM control algorithm for multilevel inverters," IEEE conf. APEC, Vol. 1, pp.562568, Feb. 2003.

[5] B. P. McGrath, D.G.Holmes, "Multi-carrier PWM strategies for multilevel inverters," IEEE Trans. Industrial Electronics, Vol. 49, pp.858-867, Aug. 2002.

[6] N. Celanovic, D. Boroyevich, "A fast space-vector modulation algorithm for multilevel three-phase converters," IEEE Trans. on Industry Applications, Vol. 37, No. 2, pp. 637 - 641, Mar./Apr. 2001.

[7] Y. Lee, D. Kim and D. Hyun, "Carrier based SVPWM method for multilevel system with reduced HDF," in Proc. IEEE IAS Annual Meeting, Rome, pp. 1996-2003, 2000.

[8] B. P. McGrath, D. G. Holmes, T. A. Lipo, "Optimised space vector switching sequences for multilevel inverters," IEEE Trans. on Power Electronics, Vol. 18, No. 6, pp. 1293 - 1301, Nov. 2003.

[9] D. G. Holmes and T. A. Lipo, Pulse width modulation for power converters - principles and practice, IEEE Press/Wiley, 2003.

[10] B. P. McGrath, D. G. Holmes, T. Meynard, "Reduced PWM harmonic distortion for multilevel inverters operating over a wide modulation range," IEEE Trans. Power Electron., Vol. 21, No. 4, pp. 941 - 949, Jul. 2006.

[11] S J.N. Chiasson, L. M. Tolbert, K J. McKenzie, and Z. Du, "A unified approach to solving the harmonic elimination equations in multilevel converters," IEEE Trans. On Power Electronics, Vol. 19, No. 2, pp. 478-490, Mar. 2004.

[12] Li Li, D. Czarkowski, Y. Liu,and P. Pillay, "Multilevel selective harmonic elimination PWM technique in series-connected voltage inverters," IEEE Trans. on Industry Applications, Vol. 36, No. 1, pp.160-169, Jan./Feb. 2000.

[13] F. Wang, "Sine-triangle vs. space vector modulation for three-level PWM voltage source inverters," IEEE Trans. on Industry Applications, Vol. 38, No. 2, pp. 500 - 506, Mar./Apr. 2002.

[14] N.V. Nho and H.H. Lee, "Carrier PWM algorithm for multi-leg multilevel inverters," in Proc. EPE, pp.1-10, Sep. 2007.

[15] N.V.Nho, M.J.Youn, "Comprehensive study on Space vector PWM and carrier based PWM correlation in multilevel invertors," IEE Proceedings Electric Power Applications, Vol.153, No.1, pp.149-158, Jan. 2006.
[16] D.W. Kang,H.C. Kim,T.J. Kim and D.S Hyun, "A simple method for acquiring the conducting angle in a multilevel cascaded inverter using step pulse waves," IEE Proc. EPA, Vol.152, No.1, 2005.

[17] T. Brückner, and D. G. Holmes, "Optimal pulse-width modulation for three-level inverters," IEEE Trans.On Power Electronics, Vol. 20, No. 1, pp. 82-89, Jan. 2005.

[18] J. Rodríguez, L. Morán, J. Pontt, P. Correa, and C. Silva, "A highperformance vector control of an 11-Level inverter," IEEE Trans. On Industrial Electronics, Vol. 50, No. 1, pp. 80-85, Feb. 2003.

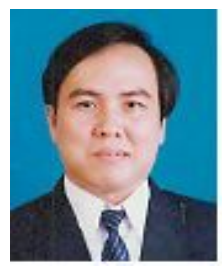

Nguyen Van Nho was born in Vietnam, in 1964. He received the M.S. and $\mathrm{PhD}$. degrees in electrical engineering from University of West Bohemia, Czech Republics in 1988 and 1991, respectively. Since 1992, he has been with Department of Electrical and Electronics Engineering, Hochiminh City University of Technology, Vietnam, where he is currently an associate professor. He was with KAIST as a post-doc fellow for six months in 2001 and a visiting professor for a year in 2003-2004. His research interests are in the areas of modeling and control of ac motors, active filters and PWM techniques.

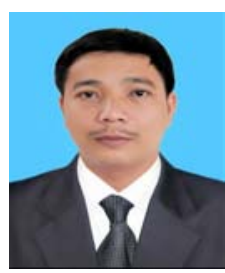

Quach Thanh Hai was born in 1972, in Vietnam. He received B.S degrees in Electrical Engineering from Hochiminh City University of Technical Education, Vietnam, in 1995 and MS degrees in Electrical Engineering from Hochiminh City University of Technology, Vietnam, in 2002. Since 1995, he has been with the Department of Electrical and Electronics Engineering, Hochiminh City University of Technical Education, Vietnam. Now, he is currently a PhD student in Electrical Engineering, Hochiminh City University of Technology, Vietnam. His research interests are power electronics and PWM techniques.

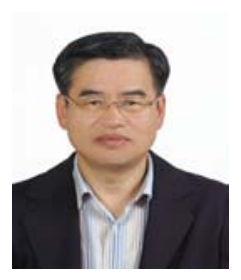

Hong-Hee Lee received his B.S., M.S., and Ph.D degrees in Electrical Engineering from Seoul National University, Seoul, Korea, in 1980, 1982, and 1990, respectively. From 1994 to 1995, he was a visiting professor with Texas A\&M University. Since 1985, he has been with the Department of Electrical Engineering, University of Ulsan, Ulsan, Korea, where he is currently a Professor of School of Electrical Engineering. He is also the Director of the Network-based Automation Research Center (NARC), which is sponsored by the Ministry of Knowledge Economy (MKE). His research interests are power electronics, network-based motor control, and control networks. He is a member of the Institute of Electrical and Electronics Engineers (IEEE), the Korean Institute of Power Electronics (KIPE), the Korean Institute of Electrical Engineers (KIEE), and the Institute of Control, Robotics and Systems (ICROS). 